\title{
FRAMEWORK IMPLEMENTATION OF THE INSPIRE ICOPE-CARE PROGRAM IN COLLABORATION WITH THE WORLD HEALTH ORGANIZATION (WHO) IN THE OCCITANIA REGION
}

\author{
N. TAVASSOLI ${ }^{1}$, A. PIAU ${ }^{1,2}$, C. BERBON ${ }^{1}$, J. DE KERIMEL ${ }^{1}$, C. LAFONT $^{1}$, P. DE SOUTO BARRETO ${ }^{1,2}$, \\ S. GUYONNET ${ }^{1,2}$, C. TAKEDA ${ }^{1}$, I. CARRIE ${ }^{1}$, D. ANGIONI ${ }^{1}$, F. PARIS ${ }^{1}$, C. MATHIEU ${ }^{1}$, P.J. OUSSET ${ }^{1}$, \\ L. BALARDY ${ }^{1}$, T. VOISIN ${ }^{1}$, S. SOURDET $^{1}$, J. DELRIEU ${ }^{1}$, V. BEZOMBES ${ }^{1}$, V. PONS-PRETRE ${ }^{1}$, \\ S. ANDRIEU ${ }^{1,2}$, F. NOURHASHEMI ${ }^{1,2}$, Y. ROLLAND ${ }^{1,2}$, M.E. SOTO ${ }^{1,2}$, J. BEARD $^{3}$, Y. SUMI $^{4}$, \\ I. ARAUJO CARVALHO ${ }^{4}$, B. VELLAS ${ }^{1,2}$
}

1. Gerontopole, W.H.O Collaborative Center for Frailty, Clinical Research and Geriatric Training, Toulouse University Hospital, 31059 Toulouse, France; 2. UPS/INSERM, UMR1027, F-31073 Toulouse, France; 3. ARC Centre of Excellence in Population Ageing Research, University of New South Wales, Sydney, Australia; 4. Department of Maternal, Newborn, Child, Adolescent Health and Ageing, World Health Organization, Geneva, Switzerland.

\begin{abstract}
Introduction: Limiting the number of dependent older people in coming years will be a major economic and human challenge. In response, the World Health Organization (WHO) has developed the «Integrated Care for Older People (ICOPE)» approach. The aim of the ICOPE program is to enable as many people as possible to age in good health. To reach this objective, the WHO proposes to follow the trajectory of an individual's intrinsic capacity, which is the composite of all their physical and mental capacities and comprised of multiple domains including mobility, cognition, vitality / nutrition, psychological state, vision, hearing. Objective: The main objective of the INSPIRE ICOPE-CARE program is to implement, in clinical practice at a large scale, the WHO ICOPE program in the Occitania region, in France, to promote healthy aging and maintain the autonomy of seniors using digital medicine. Method: The target population is independent seniors aged 60 years and over. To follow this population, the 6 domains of intrinsic capacity are systematically monitored with pre-established tools proposed by WHO especially STEP 1 which has been adapted in digital form to make remote and large-scale monitoring possible. Two tools were developed: the ICOPE MONITOR, an application, and the BOTFRAIL, a conversational robot. Both are connected to the Gerontopole frailty database. STEP 1 is performed every 4-6 months by professionals or seniors themselves. If a deterioration in one or more domains of intrinsic capacity is identified, an alert is generated by an algorithm which allows health professionals to quickly intervene. The operational implementation of the INSPIRE ICOPE-CARE program in Occitania is done by the network of Territorial Teams of Aging and Prevention of Dependency (ETVPD) which have more than 2,200 members composed of professionals in the medical, medico-social and social sectors. Targeted actions have started to deploy the use of STEP 1 by healthcare professionals (physicians, nurses, pharmacists,...) or different institutions like French National old age insurance fund (CNAV), complementary pension funds (CEDIP), Departmental Council of Haute Garonne, etc. Perspective: The INSPIRE ICOPE-CARE program draws significantly on numeric tools, e-health and digital medicine to facilitate communication and coordination between professionals and seniors. It seeks to screen and monitor 200,000 older people in Occitania region within 3 to 5 years and promote preventive actions. The French Presidential Plan Grand Age aims to largely implement the WHO ICOPE program in France following the experience of the INSPIRE ICOPE-CARE program in Occitania.
\end{abstract}

Key words: ICOPE program, older people, dependency, remote monitoring, STEP, intrinsic capacity, INSPIRE, Occitania, care implementation, clinical practice.

\section{Introduction}

The INSPIRE program was recently funded in Toulouse, France, which aims to identify the hallmarks of biological aging and allows us to propose, in the future, innovative therapeutics to prevent or restore impaired function (1). To achieve this objective, a Human Translational Cohort as well as an Animal cohort will be created to discover biomarkers of aging. The INSPIRE ICOPE-CARE program is a part of the INSPIRE program. Its main objective is to implement, at a large scale in the Occitania region, South-Western France, the WHO ICOPE program in the daily clinical routine. The ambition of INSPIRE ICOPE-CARE is to evaluate and follow about 200,000 older adults by 2025 .

WHO defines the notion of healthy aging (2), not as having no pathology since diseases happen throughout life, but as being able to keep doing what we have reason to value for as long as possible. Healthy aging partly depends on the maintenance of optimal levels of intrinsic capacity during aging, intrinsic capacity being a combination of all mental 
and physical capacities. WHO has developed the Integrated Care for Older People (ICOPE) program, a function- and person-centered care pathway during aging (3); as WHO Collaborative center for frailty, clinical research and geriatric training, the Gerontopole of Toulouse University Hospital played an important role in its elaboration. This program was created based on the analysis of more than 500 major original publications and the recommendations of a committee on aging including experts from $\mathrm{WHO}$ and almost 50 international experts from research, care and the academic world.

The ICOPE program is a care pathway, which consists of a participative and integrated healthcare approach that takes into account the individuals' capacities, their associated pathologies, the environment, their lifestyle, but also their wishes and aspirations. Emphasis is placed on the fact that the patient must be involved in his/her care and monitoring (4). In the ICOPE program, there are 5 steps in the care of the subject: STEP 1: Screen for declines in intrinsic capacity; STEP 2: Undertake a person-centered assessment in primary care; STEP 3: Define the goal of care and develop a personalized care plan; STEP 4: Ensure a referral pathway and monitoring of the care plan with links to specialized geriatric care; STEP 5: Engage community and support caregivers (Figure 1). The focus of the ICOPE program is on three important points: 1 - the patient is centrally involved in his/her care and monitoring, 2- the care plan considers the importance of caregivers and the use of local resources offered by the community; 3 - a large place is given to new technologies or "digital medicine" $(3,4)$. Indeed, information and communication technologies are crucial in the current context of medical demographic constraints, as well as the need to continuously monitor large populations.
The objective of ICOPE program is to prevent or delay the onset, and decrease the severity of care dependency. The goal is to enable as many people as possible to age in good health. To reach this objective, WHO proposes to follow the trajectory of intrinsic capacity covering six operational domains: mobility, cognition, vitality / nutrition, psychological state, vision, hearing $(5,6)$.

The large INSPIRE Program has two principal objectives (1). The first main objective is to build a resource and research platform for Geroscience extending from animals to humans, from cells to individuals, from research to clinical care. Although chronological age (civil age, date of birth) has always been used as the operational definition of aging, it does not necessarily reflect the biological process of aging. The second main objective of INSPIRE program is to implement in clinical care the WHO ICOPE Program. In the current paper, we describe the methods of implementation of the ICOPE program in the context of the INSPIRE initiative, the "INSPIRE ICOPE-CARE program".

\section{Method}

\section{Objectives of the "INSPIRE ICOPE-CARE program"}

The main objective of the INSPIRE ICOPE-CARE program is to implement, at a large scale in the Occitania region, SouthWestern France, the WHO ICOPE program in a daily clinical routine. Secondary objectives are to explore the acceptability of the INSPIRE ICOPE-CARE program by both older adults and health care professionals, as well as to examine the use of new digital tools in the evaluation and monitoring of intrinsic capacity.

Figure 1

5 Steps of WHO ICOPE program

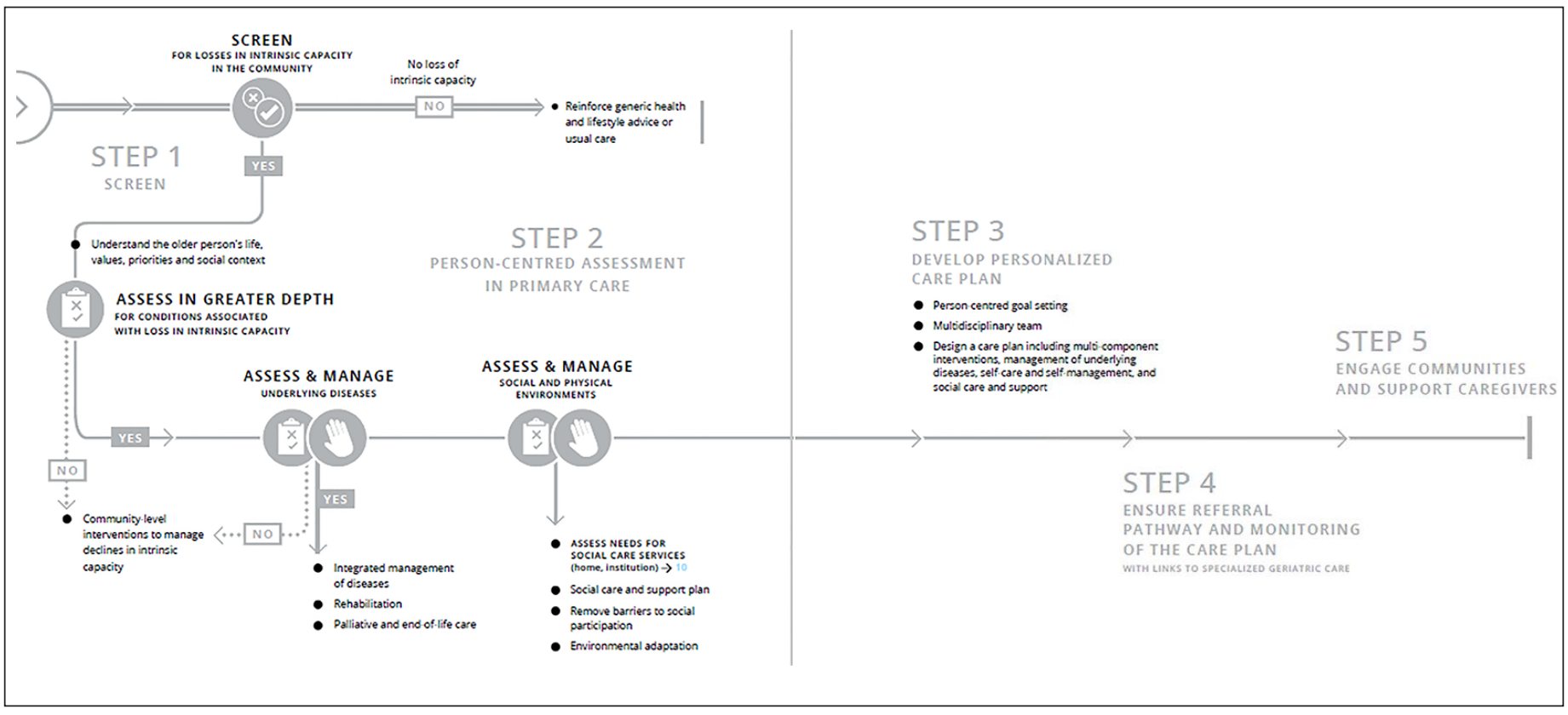




\section{THE JOURNAL OF FRAILTY \& AGING}

\section{Population}

The target population in the INSPIRE ICOPE-CARE program is independent seniors aged 60 years and over in Occitania region, in France. The actors are healthcare professionals, trained professionals, but also caregivers and seniors themselves. Indeed, the STEP 1 tool may be used by any person who has undergone a training course on ICOPE. To follow this population, the six domains of intrinsic capacity are systematically monitored with pre-established tools (STEP 1, and then if appropriate STEP 2, STEP 3, STEP 4, STEP 5). This allows the health professionals to intervene quickly if a decline occurs in any domain.

\section{STEP 1 - Screen for declines in intrinsic capacity}

The first tool proposed by WHO to evaluate intrinsic capacity is a screening tool referred to as ICOPE STEP 1 . It is very simple, and usable by actors who are not necessarily health professionals after a brief training course (Figure 2). It allows a rapid assessment of the six operational domains of intrinsic capacity by very simple tests. In the INSPIRE ICOPECARE program, this tool has been adapted by the Toulouse Gerontopole to become a tool capable of monitoring intrinsic capacity over time. Then, the adapted ICOPE STEP 1 was elaborated in digital format to make it possible to undertake remote monitoring in a large-scale.

Figure 2

WHO STEP 1 screening tool (from WHO with permission)

\begin{tabular}{|c|c|c|}
\hline $\begin{array}{l}\text { Priority conditions associated } \\
\text { with declines in intrinsic capacity }\end{array}$ & Tests & $\begin{array}{l}\text { Assess fully if any answer } \\
\text { in each domain triggers this }\end{array}$ \\
\hline \multirow{3}{*}{$\begin{array}{l}\text { COGNITIVE DECLINE } \\
\text { (Chapter 4) }\end{array}$} & 1. Remember three words: flower, door, rice (for example) & \\
\hline & $\begin{array}{l}\text { 2. Orientation in time and space: What is the full date today? } \\
\text { Where are you now (home, clinic, etc)? }\end{array}$ & $\begin{array}{l}\text { Wrong to either } \\
\text { question or does } \\
\text { not know }\end{array}$ \\
\hline & 3. Recalls the three words? & $\begin{array}{l}\text { Cannot recall all } \\
\text { three words }\end{array}$ \\
\hline $\begin{array}{l}\text { LIMITED MOBILITY } \\
\text { (Chapter 5) }\end{array}$ & $\begin{array}{l}\text { Chair rise test: Rise from chair five times without using arms. } \\
\text { Did the person complete five chair rises within } 14 \text { seconds? }\end{array}$ & No \\
\hline \multirow[t]{2}{*}{$\begin{array}{l}\text { MALNUTRITION } \\
\text { (Chapter 6) }\end{array}$} & $\begin{array}{l}\text { 1. Weight loss: Have you unintentionally lost more than } 3 \mathrm{~kg} \\
\text { over the last three months? }\end{array}$ & Yes \\
\hline & 2. Appetite loss: Have you experienced loss of appetite? & Yes \\
\hline $\begin{array}{l}\text { VISUAL IMPAIRMENT } \\
\text { (Chapter 7) }\end{array}$ & $\begin{array}{l}\text { Do you have any problems with your eyes: difficulties in } \\
\text { seeing far, reading, eye diseases or currently under } \\
\text { medical treatment (e.g.g diabetes. high blood pressure)? }\end{array}$ & Yes \\
\hline \multirow{3}{*}{$\begin{array}{l}\text { HEARING LOSS } \\
\text { (Chapter 8) }\end{array}$} & Hears whispers (whisper test) or & \\
\hline & Screening audiometry result is $35 \mathrm{~dB}$ or less or & Fail \\
\hline & Passes automated app-based digits-in-noise test & \\
\hline \multirow{3}{*}{$\begin{array}{l}\text { DEPRESSIVE SYMPTOMS } \\
\text { (Chapter 9) }\end{array}$} & Over the past two weeks, have you been bothered by & Yes \\
\hline & - feeling down, depressed or hopeless? & 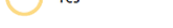 \\
\hline & - little interest or pleasure in doing things? & Yes \\
\hline
\end{tabular}

The mobile phone is the technology that has spread best among the older population and is therefore an ideal support to remotely screen or monitor health indicators (7). Thus, two tools were developed by the Toulouse Gerontopole: a) the ICOPE MONITOR, an application, which is an adapted version of the ICOPE WHO application, accessible via smartphone or tablet; and b) the BOTFRAIL, an internet conversational robot, accessible via computer, smartphone or tablet. These two complementary tools can be used in two modes: professional mode and self-assessment mode for the senior or his/her caregiver. Both are connected to the pre-existing Gerontopole frailty database created in 2016 to collect medical and sociodemographic data from frail older people who received a face to face standardized gerontological assessment in the Occitania region. Data from more than 6,000 patients in 180 different health centers have already been collected since 2017. The database complies with all French and European regulations in terms of health data security. The authorization of the French "National Commission for Data Protection" was granted on April 13, 2017 (Ref. Nb. MMS/OSS/NDT171027, authorization request $\mathrm{Nb}$. 19141154).

These tools may be used by everyone. However, in the context of the INSPIRE ICOPE-CARE program, during the first (face to face) STEP 1 screening, the professional collects the senior's oral consent to keep his/her data in the frailty database as well as to monitor his/her intrinsic capacity regularly. If the assessment is normal, lifestyle advice is provided by the professional who also trains the senior or his family/caregiver to use the tools in «self-assessment» mode.

\section{Figure 3}

Diagram of the INSPIRE ICOPE-CARE program

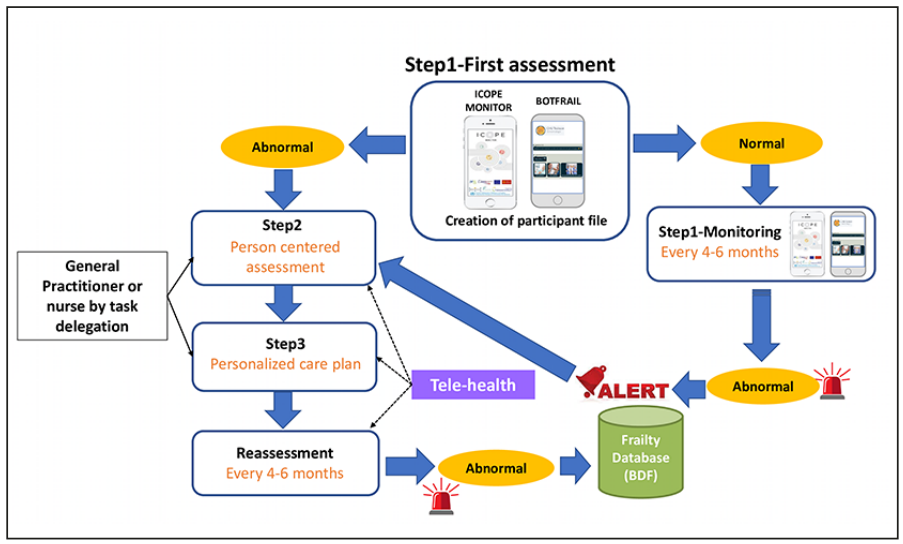

Remote screening of STEP 1 - ongoing monitoring

The ongoing screening of intrinsic capacity deficits is based on a remote monitoring system. After having been taught by health professionals how to use the ICOPE MONITOR app and/ or BOTFRAIL tool, seniors are invited to continuously use the STEP 1 every 4-6 months. If the senior cannot carry out the self-assessment, a professional will intervene every 6 months to perform the STEP 1 follow-up. STEP 1 data collected using the ICOPE MONITOR application or BOTFRAIL every 4 to 6 months are transmitted to the Gerontopole frailty database. If a deterioration in one or more domains of intrinsic capacity is identified during monitoring, an alert is generated by an algorithm. During the first deployment phase of the project, the management and processing of these alerts is carried out by the experienced nurses of the Gerontopole in collaboration with the primary care providers [General Practitioners (GP) and nurses]. 
Table 1

Tele-health supports and INSPIRE ICOPE-CARE program

\begin{tabular}{|c|c|c|c|}
\hline & STEP 1 & STEP 2 / STEP 3 & STEP 4 \\
\hline Face to face & $\begin{array}{l}\text { First assessment / Health profes- } \\
\text { sional }\end{array}$ & $\begin{array}{l}\text { Assessment by the nurse/GP if } \\
\text { STEP } 1 \text { is abnormal or if an alert } \\
\text { is generated during follow-up }\end{array}$ & - \\
\hline $\begin{array}{l}\text { Remote monitoring and support, } \\
\text { dashboard (alerts) }\end{array}$ & $\begin{array}{l}\text { Time repetition of STEP } 1 \text { every } \\
4-6 \text { months / self-assessment }\end{array}$ & - & $\begin{array}{l}\text { a. Time repetition of STEP } 1 \text { eve- } \\
\text { ry } 4-6 \text { months / self-assessment } \\
\text { b. Strengthening adherence to the } \\
\text { care plan / nurse } \\
\text { c. Care coordination / nurse }\end{array}$ \\
\hline Tele-consultation (Nurse, GP, G) & - & $\begin{array}{l}\text { Assessment by the nurse/GP if } \\
\text { STEP } 1 \text { is abnormal or if an alert } \\
\text { is generated during follow-up }\end{array}$ & $\begin{array}{l}\text { a. Time repetition of STEP } 1 \text { eve- } \\
\text { ry } 4-6 \text { months / nurse support } \\
\text { b. Teleconsultation with the } \\
\text { reference center (e.g. geriatrician, } \\
\text { psychiatrist, nutritionist) }\end{array}$ \\
\hline
\end{tabular}

Note. The follow-up is always digital (self-assessment / caregiver or nurse support). Depending on the geographical context and health resources, STEP 2 can be performed face-to-face or through telemedicine tools. The actors involved are also context-dependent; Note: Tele -consultation is a tele-health consultation between a doctor/nurse and the senior. Tele expertise

is between two health professionals; General Practitioner, GP, geriatricians, G.

When an alert is detected, the senior or family (as appropriate) will be contacted to verify the clinical relevance of the intrinsic capacity deficit. If the deficit is confirmed, the GP will be contacted to initiate STEP 2. This first phase makes it possible to evaluate the relevance of the alerts, adjust the algorithm and develop decision trees (Figure $3 \&$ Table 1).

STEP 2 and STEP 3 - Undertake a person-centered assessment in primary care, define the goal of care and develop a personalized care plan

If the screening of intrinsic capacity is abnormal, STEP 2 (person-centered assessment) and STEP 3 (personalized care plan) are carried out by the GP or, if possible, by a nurse trained in geriatrics using the delegation of task as part of the "French protocol of cooperation" or the «Hospital outside the walls» care unit, as described below:

- The cooperation protocol was developed by the Gerontopole of Toulouse and the Occitania Regional Health Agency (ARS) and obtained the approval from the High Authority for Health (HAS) on December 4, 2013 (8, 9 ). It aims to delegate to a self-employed trained nurse (40 hours of training), the geriatric assessment of older people identified as frail. At the end of this assessment, the nurse, can immediately refer the person to the GP (in the case of warning signs or unexplained or multiple anomalies), to social services, appropriate health professionals (specialist physicians, physiotherapists,...) or themselves initiate preventive measures. The delegated nurse intervenes at the request of the GP after obtaining the consent of the patient. The nurse's evaluations and proposed interventions are re-analyzed and discussed during a multi-professional concertation meeting with patient's GP, organized no later than 30 days following the geriatric assessment. This model of care is funded by local health authorities. To date, more than 160 nurses have been trained in the Occitania region.

- The «Hospital outside the walls» care unit of Gerontopole is an innovative unit of care created in 2015 at the Toulouse University Hospital to take care of frail older people outside the hospital. The geriatric evaluation of the older people is carried out by experienced trained nurses of the Gerontopole at their homes or in public spaces close to their homes. These nurses, with the support of a hospital geriatrician, propose a personalized care plan to the older people evaluated. This plan is then sent to the person's GP who ensures its implementation and follow-up. This model has been rolled out in other hospital centers in the Occitania region. To date seven hospitals in the region have their own «Hospital outside the walls» care unit.

STEP 4 - Ensure a referral pathway and monitoring of the care plan with links to specialized geriatric care

As mentioned above, monitoring consists of the repetition every 4-6 months of STEP 1, either by self-assessment or with the help of a family member or professional caregiver. Digital medicine used in the INSPIRE ICOPE-CARE program makes it possible to simultaneously monitor large populations regardless of where they live and the extent of local medical resources and can also ensure the implementation and follow up of the personalized care plan proposed in STEP 3. Thus, digital medicine allows to focus the action of the health professionals on those who need and when they 


\section{THE JOURNAL OF FRAILTY \& AGING}

need (efficient use of resources and personalized medicine) using a dashboard and by the generation of automatized and graduated alerts (the algorithm will evolve over time). When a coordination meeting is necessary between the GP and other health professionals especially nurses who realized STEP2 and STEP3, tele-health consultations may be used (called tele-expertise). To make it possible, we are deploying the use of a tele-health platform to facilitate access to this expertise throughout the region regardless of location. In the context of "complexes cases", the GP may request a tele-consultation or tele-expertise with geriatricians and other physicians (e.g. psychiatrists) in the reference center. In these situations all the Gerontopole's paramedical resources: physiotherapists, nutritionists, neuropsychologists and social workers may be involved, as well.

Lastly, digital remote monitoring tools are an excellent support for assessing adherence to the care plan, coordinate care, but also for providing information or educational content (nutrition, physical activity) to seniors.

\section{STEP 5 - Engage community and support caregivers}

This stage allows the establishment of an ecosystem favorable to «healthy aging». At this step, town halls, departments, and regions as well as several organizations (Departmental Councils, pension funds, insurances,...) can set up efficient organizations to encourage and promote «healthy aging». The INSPIRE ICOPE-CARE program is part of this step and plays an important role in bringing together different actors and organizations around the same objective which is the adaptation of our society to aging well.

Implementation of concrete actions in the Occitania region

The operational implementation of the INSPIRE ICOPECARE program in Occitania will be promoted by the network of Territorial Teams of Aging and Prevention of Dependency (ETVPD) which have more than 2,200 members composed of professionals in the medical, medico-social and social sectors. This Network was created in 2012 by the Gerontopole of Toulouse with the support of the Occitania Regional Health Agency (ARS). The objective of this network is to prevent dependency among seniors in Occitania by promoting care, training, research and innovation in gerontology. Several meetings take place every year in different territories in Occitania allowing to exchange with the actors of the field and to advance on innovative projects. The INSPIRE ICOPE-CARE program is currently the main project on which the territorial teams are working.

A number of targeted actions have started to facilitate the use of STEP 1 by healthcare professionals or different institutions:

Physicians: The GP has the principal role for the implementation of the intervention plan proposed to the senior in the INSPIRE ICOPE-CARE program. His/her adherence to the program is essential. We are currently working with the regional union of physicians and the University Department of
General Medicine (DUMG) to establish a follow-up strategy adapted to the GP's work.

Nurses: In the INSPIRE ICOPE-CARE program, the nurse, in connection with the GP, will coordinate the implementation of intervention plans. First, we started the implementation of the INSPIRE ICOPE-CARE program with our experienced nurses of the Gerontopole who work at the «Hospital outside the walls» care unit of Toulouse University Hospital. Since January 2019, these nurses have used the STEP 1 tool for all their patients; to date (March 2020), around 950 seniors have been assessed. Some preliminary data on step 1 in 755 subjects showed that mean age was $80.9 \pm 7.3$ years, $67.3 \%$ were female $(n=298)$ and $699(92.6 \%)$ had at least one domain of intrinsic capacity affected. Table 2 shows the results of the STEP 1 evaluations.

The nurses especially those trained on frailty assessment in the framework of cooperation protocol (more than 160 nurses) are also involved. Since January 2020, the Gerontopole has organized several training courses on the use of STEP 1 and ICOPE. To date, 94 nurses have been trained and 240 are registered for the next sessions. An agreement was signed between the Toulouse University Hospital and the Occitania Regional Health Agency to pay nurses 15 euros for this evaluation.

Table 2

STEP 1 analysis performed on the first 755 subjects evaluated

\begin{tabular}{lc}
\hline Characteristics & Mean \pm SD or n (\%) \\
\hline Age (years); $n=442$ & $80.9 \pm 7.3$ \\
Gender (female); $=443$ & $298(67.3)$ \\
Mobility (chair rise test) & \\
Test not feasible; $\mathrm{n}=740$ & $96(13.0)$ \\
Time to do 5chair rises (seconds); $=644$ & $13.6 \pm 5.4$ \\
Abnormality in mobility domain; $\mathrm{n}=749$ & $333(44.5)$ \\
Cognition (3 words recall and orientation) & \\
Abnormality in cognition domain; $\mathrm{n}=746$ & $362(51.7)$ \\
Psychology (depression symptoms) & \\
Abnormality in psychology domain; $\mathrm{n}=743$ & $250(33.6)$ \\
Vision (eye problems) & \\
Abnormality in vision domain; $\mathrm{n}=737$ & $430(58.3)$ \\
Hearing (whisper test) & \\
Abnormality in hearing domain; $\mathrm{n}=747$ & $313(41.9)$ \\
Vitality/nutrition (weight or appetite loss) & \\
Abnormality in nutrition domain; $\mathrm{n}=741$ & $124(16.7)$ \\
\hline
\end{tabular}

Pharmacists: In collaboration with the regional union of pharmacists and the pharmacy unit of Toulouse University Hospital, volunteer pharmacists and 6th year pharmacy students are also trained on the ICOPE program. To date (March 2020), 
65 pharmacists have been trained and approximately 80 are registered for the next training sessions. The STEP 1 will be performed in the pharmacies by the pharmacists and in the event of an abnormal STEP 1, the pharmacist will direct the patient to his/her GP.

Institutions: A partnership is set up with CEDIP (CEntre D'Information et de Prévention - Agirc-Arrco), which is a complementary pension fund in France, to carry out STEP 1 for their beneficiaries. To date (March 2020), more than 300 seniors have been assessed. An analysis made on 207 subjects with usable data showed that mean age was 70.1 years, $61.8 \%$ were female $(n=128)$ and $86.5 \%(n=179)$ had at least one domain of intrinsic capacity affected. The domain most affected was vision $(64.7 \%)$ followed by hearing (60.9\%) and cognition $(41.5 \%)$.

A collaboration is developed with French National old age insurance fund (CNAV) to implement STEP 1 within the usual professional practices of home caregivers and create an innovative and specific prevention offer for young precarious retirees (62-70 years old).

Departmental Council of Haute Garonne is a partner of the Gerontopole of Toulouse. Their assessors are planned to be trained in June 2020 to carry out STEP 1 for all independent seniors who request them for a personalized autonomy allowance.

Several other actions are planned. A project is being developed with the French Mutual Insurance, which brings together the majority of existing mutual health insurance companies in France, to disseminate information about this program to primary healthcare providers. The Federation of Health Homes of Occitania (FORMS) was contacted to set up the INSPIRE ICOPE-CARE program in Health Homes. We are also collaborating with the Post Office, to set up an experiment in three cities in the Toulouse agglomeration in order to carry out STEP 1 by trained postmen from the Post Office. The experiment is due to start in September 2020. We are also working with spas, which receive a large number of seniors each year.

INSPIRE ICOPE-CARE program: perspectives and future challenges for the care of individuals during aging

It is always difficult to change habits and implement new care pathways in clinical practice. The INSPIRE ICOPE-CARE program plans to screen and monitor the intrinsic capacity of 200,000 older people in Occitania region within five years and promote preventive actions, instead of only punctual, curative ones. WHO with ICOPE program is determined to reduce the number of older people worldwide who are care dependent by 15 million by 2025 , which would mean 150,000 in France and 15,000 in Occitania (10).

The INSPIRE ICOPE-CARE program is in full agreement with the national project of "Ma santé 2022" (My Health 2022) (11), in which the French Ministry of Health highlights the following aspects: to organize healthcare around the older people and give them a qualified and relevant care, be more active in prevention in order to promote home maintenance and develop a better organization of care between healthcare providers with the support of digital medicine. In order to achieve these objectives, the nurses' role has to be redefined, giving them a stronger place in the assessment and the coordination of the patient's healthcare pathway. Another change has to take place in communication and organization between hospital and primary healthcare providers, especially GPs. To establish this new organization, the INSPIRE ICOPECARE program supports "Ma Santé 2022" by joining the projects of several Professional regional health communities (CPTS) in the Occitania region. The CPTS are a new mode of organization that allows health professionals to come together in the same territory around a common medical and medicosocial project. Moreover, The French Presidential Plan Grand Age aims to largely implement the WHO ICOPE program in France following the experience of the INSPIRE ICOPE-CARE program in Occitania.

This initiative draws significantly on numeric tools, e-health and digital medicine to facilitate communication and coordination between professionals and seniors $(12,13)$, it plays an important role in the future of geriatrics (14-17). The INSPIRE ICOPE-CARE program will also allow us to implement in clinical practice the discoveries of INSPIRE platform concerning clinical and biological biomarkers (18-22).

Funding: The Inspire Program was supported by grants from the Region Occitanie/Pyrénées-Méditerranée (Reference number: 1901175), the European Regional Development Fund (ERDF) (Project number: MP0022856), ARS «Agence Régional de Santé d'Occitanie» and the Inspire Chairs of Excellence funded by: Alzheimer Prevention in Occitania and Catalonia (APOC), EDENIS, KORIAN, Pfizer, Pierre-Fabre, Fondation Avenir Cogfrail Grant.

Potential Conflicts of Interest: The authors declare that they have no conflict of interest for the present paper.

Acknowledgements: The authors thank all the health professionals participating in the INSPIRE ICOPE CARE Program especially Cendrine Blazy and Dr Marie Dominique Medou from Occitania Regional Health Agency and all the members of the Gerontopole "Hospital of the walls" care unit (Augusto S, Bouchon L, Cazes MC, Da Costa F, Poly M, Vaysset S) and all the members of the Occitania Territorial Teams of Aging and Prevention of Dependency.

Ethical standards: The INSPIRE protocol has been approved by the French Ethical Committee located in Rennes (CPP Ouest V) in October 2019. This research has been registered on the site http://clinicaltrials.gov (ID NCT04224038). In the INSPIRE ICOPE-CARE program, all the senior's data are collected in the Gerontopole Frailty database. This database complies with all French and European regulations in terms of health data security. The authorization of the French "National Commission for Data Protection" was granted on April 13, 2017 (Ref. Nb. MMS/OSS/ NDT171027, authorization request Nb. 19141154). During the first (face to face) STEP 1 screening, the professional collects the 


\section{THE JOURNAL OF FRAILTY \& AGING}

senior's oral consent to keep his/her data in the frailty database as well as to monitor his/her intrinsic capacity regularly.

Open Access: This article is distributed under the terms of the Creative Commons Attribution 4.0 International License (http://creativecommons.org/licenses/by/4.0/), which permits use, duplication, adaptation, distribution and reproduction in any medium or format, as long as you give appropriate credit to the original author(s) and the source, provide a link to the Creative Commons license and indicate if changes were made.

Corresponding author: Neda Tavassoli, Équipe Régionale Vieillissement et Prévention de la Dépendance (ERVPD), La Cité de la Santé, Bâtiment Ex-Biochimie, Hôpital La Grave, Place Lange, TSA 60033, 31059 Toulouse Cedex 9, France, Phone number: +33561 7770 13, Fax number: +33561 7764 75, E-mail address: tavassoli.n@chu-toulouse.fr

\section{References}

1. De Souto Barreto P, Guyonnet S, Ader GI, Andrieu S, Casteilla L, Davezac N, Dray C, Fazilleau N, Gourdy P, Liblau R, Parini A, Payoux P. The INSPIRE research initiative: A program for geroscience and healthy aging research going from animal models to humans and the healthcare system. J Frailty Aging 2020;Doi: 10.14283/ jfa.2020.18.

2. Beard JR, Jotheeswaran AT, Cesari M, Araujo de Carvalho I. The structure and predictive value of intrinsic capacity in a longitudinal study of ageing. BMJ Open. 2019;9(11):e026119.

3. WHO Guidelines on Integrated Care for Older People (ICOPE). WHO. Available at: https://apps.who.int/iris/bitstream/handle/10665/326843/WHO-FWC-ALC-19.1-eng. pdf;jsessionid=31CB3214293723D1D9A7D2B822B92D0E? sequence=1 $($ accessed April 2, 2020).

4. Islene Araujo de Carvalho, a JoAnne Epping-Jordan,b Anne Margriet Pot,c Edward Kelley,d Nuria Toro,e Jotheeswaran A Thiyagarajana \& John R Beard. Organizing integrated health-care services to meet older people's Needs Bull World Health Organ 2017;95:756-763.

5. Cesari M, Araujo de Carvalho I, Amuthavalli Thiyagarajan J, Cooper C, Martin FC, Reginster JY, Vellas B, Beard JR. Evidence for the Domains Supporting the Construct of Intrinsic Capacity. J Gerontol A Biol Sci Med Sci. 2018;73(12):1653-1660.

6. Beard JR, Jotheeswaran AT, Cesari M, Araujo de Carvalho I. The structure and predictive value of intrinsic capacity in a longitudinal study of ageing. BMJ Open. 2019;9(11):e026119.

7. Mobile Fact Sheet. Pew Research Center Internet \& American Life Project. Available at: http://www.pewinternet.org/fact-sheet/mobile/ (accessed April 2, 2020).

8. AVIS $\mathrm{N}^{\circ}$ 2013.0092/AC/SEVAM du 4 décembre 2013 du collège de la Haute Autorité de santé relatif au protocole de coopération «Intervention d'infirmières libérales à domicile afin de diagnostiquer et d'initier la prise en charge de la fragilité du sujet âgé». Available at: https://www.has-sante.fr/upload/docs/application/pdf/201403/a_2013_0092_pc_046.pdf (accessed April 2, 2020).
9. Piau A, Sourdet S, Toulza O, Bernon C, Tavassoli N, Nourhashemi F. Frailty Management in Community-Dwelling Older Adults: Initial Results of a Trained Nurses Program. J Am Med Dir Assoc. 2019;20(5):642-643.

10. WHO Clinical Consortium on Healthy Ageing 2018, Geneva, Switzerland. Available at: https://apps.who.int/iris/bitstream/handle/10665/330026/WHO-FWC-ALC-19.2 eng.pdf (accessed April 2, 2020).

11. Ma santé 2022, un engagement collectif. May 3, 2019. Available at: https://solidaritessante.gouv.fr/IMG/pdf/dossier_de_presse_acces_aux_soins_avril2019_vdef.pdf (accessed April 2, 2020).

12. Takeda C., Guyonnet S., Sumi Y. Vellas B. Integrated Care for Older People and the Implementation in the INSPIRE Study. J Prev Alz Dis 2020;2(7):70-74

13. Beard J.R. Linking Geroscience and Integrated Care to Reinforce Prevention. J Prev Alz Dis 2020;2(7):68-69.

14. Morley JE. Editorial: The Future of Geriatrics. J Nutr Health Aging.2020;24(1):1-2 doi: 10.1007/s12603-019-1308-5. PubMed PMID: 31886800.

15. Marengoni A. Letter to the editor: Reply to: The Future of Geriatrics. J Nutr Health Aging. 2020;24(2):242. doi: 10.1007/s12603-020-1321-8. PubMed PMID:32003418

16. Vellas B, Scrase D, Rosenberg GA, Andrieu S, Araujo de Carvalho I, Middleton LT. Editorial: WHO Guidelines on Community-Level Interventions to Manage Declines in Intrinsic Capacity: The Road for Preventing Cognitive Declines in Older Age? J Prev Alzheimers Dis. 2018;5(3):165-167.

17. Dent E, Morley JE, Cruz-Jentoft AJ, Woodhouse L, Rodríguez-Mañas L, Fried LP, Woo J, Aprahamian I, Sanford A, Lundy J, Landi F, Beilby J, Martin FC, Bauer JM, Ferrucci L, Merchant RA, Dong B, Arai H, Hoogendijk EO, Won CW, Abbatecola A,Cederholm T, Strandberg T, Gutiérrez Robledo LM, Flicker L, Bhasin S, AubertinLeheudre M, Bischoff-Ferrari HA, Guralnik JM, Muscedere J, Pahor M, Ruiz J, Negm AM, Reginster JY, Waters DL, Vellas B. Physical Frailty: ICFSR International Clinical Practice Guidelines for Identification and Management. J Nutr Health Aging. 2019;23(9):771-787. doi: 10.1007/s12603-019-1273-z. PubMed PMID: 31641726; PubMed Central PMCID: PMC6800406.

18. Guerville F, De Souto Barreto P, Ader I, Andrieu S, Casteilla L, Dray C, Fazilleau N Guyonnet S, Langin D, Liblau R, Parini A, Valet P, Vergnolle N, Rolland Y, Vellas B. Revisiting the Hallmarks of Aging to Identify Markers of Biological Age. J Prev Alzheimers Dis. 2020;7(1):56-64.

19. Sierra F. Editorial: Geroscience and the Role of Aging in the Etiology and Management of Alzheimer's Disease. J Prev Alzheimers Dis. 2020;7(1):2-3.

20. Berg-Weger M, Morley J. Editorial: Loneliness in Old Age: An unaddressed Health Problem. J Nutr Health Aging. 2020;24(3):243-245. doi: 10.1007/s12603-020-1323-6. PubMed PMID: 32115602 .

21. Guralnik J, Bandeen-Roche K, Bhasin SAR, Eremenco S, Landi F, Muscedere J, Perera S, Reginster JY, Woodhouse L, Vellas B. Clinically Meaningful Change for Physical Performance: Perspectives of the ICFSR Task Force. J Frailty Aging 2020;9(1):9-13. doi: 10.14283/jfa.2019.33. PubMed PMID: 32150208

22. Rodriguez-Mañas L, Araujo de Carvalho I, Bhasin S, Bischoff-Ferrari HA, Cesari M, Evans W, Hare JM, Pahor M, Parini A, Rolland Y, Fielding RA, Walston J, Vellas B ICFSR Task Force Perspective on Biomarkers for Sarcopenia and Frailty. J Frailty Aging. 2020;9(1):4-8. doi: 10.14283/jfa.2019.32. PubMed PMID: 32150207. 